\title{
Vitamin D receptor expression in invasive breast tumors and breast cancer survival
}

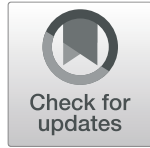

\author{
Linnea Huss ${ }^{1 *}$ (D), Salma Tunå Butt ${ }^{1}$, Signe Borgquist ${ }^{2}$, Karin Elebro ${ }^{3}$, Malte Sandsveden ${ }^{1}$, Ann Rosendah ${ }^{3 \dagger}$ and \\ Jonas Manjer ${ }^{1+}$
}

\begin{abstract}
Background: Vitamin D has been suggested to prevent and improve the prognosis of several cancers, including breast cancer. We have previously shown a U-shaped association between pre-diagnostic serum levels of vitamin D and risk of breast cancer-related death, with poor survival in patients with the lowest and the highest levels respectively, as compared to the intermediate group. Vitamin D exerts its functions through the vitamin D receptor (VDR), and the aim of the current study was to investigate if the expression of VDR in invasive breast tumors is associated with breast cancer prognosis.
\end{abstract}

Methods: VDR expression was evaluated in a tissue microarray of 718 invasive breast tumors. Covariation between VDR expression and established prognostic factors for breast cancer was analyzed, as well as associations between VDR expression and breast cancer mortality.

Results: We found that positive VDR expression in the nuclei and cytoplasm of breast cancer cells was associated with favorable tumor characteristics such as smaller size, lower grade, estrogen receptor positivity and progesterone receptor positivity, and lower expression of Ki67. In addition, both intranuclear and cytoplasmic VDR expression were associated with a low risk of breast cancer mortality, hazard ratios $0.56(95 \% \mathrm{Cl} 0.34-0.91)$ and $0.59(0.30-1.16)$ respectively.

Conclusions: This study found that high expression of VDR in invasive breast tumors is associated with favorable prognostic factors and a low risk of breast cancer death. Hence, a high VDR expression is a positive prognostic factor.

Keywords: Vitamin D receptor, Breast cancer, Survival, Mortality, Tissue microarray

\section{Introduction/background}

An enlarging body of research suggests that relatively low levels of vitamin D are associated with a poor breast cancer prognosis [1-4]. In the only study to date using pre-diagnostic levels of vitamin D, we found that women with high vitamin $\mathrm{D}$ levels were also at high risk of breast cancer death, compared to women with intermediate levels [5].

Vitamin D exerts its function through the vitamin D receptor (VDR), a nuclear receptor that modulates transcription of target genes, [6] and is to be found in lobule and ductal epithelial cells in normal mammary glands $[7,8]$.

\footnotetext{
* Correspondence: Linnea.Huss@med.lu.se

${ }^{\dagger}$ Ann Rosendahl and Jonas Manjer contributed equally to this work.

1 Department of Surgery, Lund University, Skåne University Hospital, SE-205

02 Malmö, Sweden

Full list of author information is available at the end of the article
}

Compared to normal breast tissue, breast cancer lesions have been found to express more VDR [9]. Since women with intermediate vs low levels of vitamin $\mathrm{D}$ may have a better survival following breast cancer, it could be assumed that VDR expression in breast tumors is also associated with a better prognosis.

Only a few studies have reported on breast cancer VDR expression in relation to tumor prognostic factors and breast cancer survival. These studies have shown differing results [10-15], and most of them investigated a rather limited number of breast tumors. The largest study to date showed associations with some tumor-related prognostic factors, but not with survival [10].

In the present study, immunohistochemical staining of VDR was performed on over 700 primary, invasive, breast tumors from the Malmö Diet and Cancer Study 
(MDCS) [16]. VDR expression was studied in relation to established tumor-related prognostic factors and breast cancer-specific mortality. The hypothesis was that breast cancers with VDR expression would be associated with less aggressive tumors and a low risk of breast cancer death, i.e., a better survival.

\section{Material and methods}

\section{The Malmö Diet and Cancer Study (MDCS)}

The Malmö Diet and Cancer Study is a prospective cohort study which during the time period 1991-1996 included citizens of Malmö, the third largest city in Sweden. All women born between 1923 and 1950 were invited to participate, and $43 \%$ of eligible women completed baseline examinations and a questionnaire about socioeconomic factors, previous disease, and medications. Eventually, 17,035 women were included in the cohort and written informed consent was obtained from all participants. The ethical committee in Lund, Sweden, approved the MDCS (LU 51-90) and the present study (Dnr 652/2005 and Dnr 23/2007).

\section{Study population}

Women included in the MDCS were followed using the Swedish Cancer Registry until December 31, 2010, and the Swedish cause of death registry up until December 31, 2016. Since 576 women out of the 17035 had already been diagnosed with breast cancer prior to baseline examination, these women were excluded from the present study. During the follow-up until December 31, 2010, 1018 women were diagnosed with breast cancer. The intention was to investigate tumor characteristics in relation to breast cancer mortality, and due to this, 68 patients with cancer in situ were excluded, since these tumors are associated with a very low breast cancer mortality, if any. Also, patients who had received neoadjuvant treatment $(n=4)$, had distant metastases at diagnosis $(n=14)$, or died from breast cancer-related causes within less than 0.3 years from diagnosis $(n=2)$ were excluded, as well as one woman who declined treatment for 4 years. Bilateral cases $(n=17)$ were also excluded due to difficulties in interpreting tumor characteristics. The final study population consisted of 912 patients (Fig. 1).

\section{Histopathological analysis and clinical information}

Medical records and pathological reports were used to retrieve information on size and laterality of tumors as well as axillary lymph node involvement. Invasive tumors diagnosed during 1991-2004 were pathologically re-evaluated as regards to invasiveness, histological type, grade, estrogen receptor (ER) status, and progesterone receptor (PgR) status by one senior pathologist $[17,18]$. Such information was readily available from pathological reports dating 2005 and onwards, with no need for reevaluation. Information on human epidermal growth factor-2 (HER2) as well as proliferation index (Ki67) on tumors dating 1991-2007 was assessed using tissue microarrays (TMAs) [19]. From 2008 and onwards, information on HER2 and Ki67 status was also retrieved from the diagnostic pathology report. ER and PgR were considered positive at a cut-off of $>10 \%$ positively stained nuclei. Results from in situ hybridization (ISH) were used to define HER2 status when available. When immunohistochemistry (IHC) evaluation was used to define HER2 status, HER2 was considered positive when annotated $3+$ and negative for 0 or $1+$. IHC scores of $2+$ were categorized as missing if ISH was not used to confirm the result [16]. For Ki67, the distribution was noted to differ between tumors diagnosed at different periods of time. Therefore, tumors were classified into low, intermediate, or high Ki67 expression based on tertiles within the diagnostic period: 1991-2004, 2005-2007, and from 2008 and onwards.

Based on histological grade; ER, PgR, and HER2 status; and Ki67 category, tumors were classified into molecular subtypes: Luminal A-like, Luminal B-like, HER2 positive, and triple negative, according to criteria used locally within the south Swedish health care region [20]. Luminal A-like tumors were defined as ER positive, HER2 negative, and either (a) histological grade 1, (b) histological grade 2 and low Ki67, or (c) histological grade 2, intermediate Ki67, and positive PgR status. Luminal B-like tumors were also ER positive and HER2 negative but associated with either (a) histological grade 3, (b) histological grade 2 and high Ki67, or (c) histological grade 2, intermediate Ki67, and negative PgR. Regardless of histological grade and hormone receptor status, all HER2-positive tumors were categorized as HER2 positive. All tumors considered ER negative, PgR negative, and HER2 negative were classified as triple negative (ibid.).

Clinical notes were used to retrieve information on the type of breast surgery, surgery to the axillary lymph nodes, and planned adjuvant therapy, as recommended by a multidisciplinary treatment conference following surgery.

\section{Vitamin D receptor expression}

Tumors diagnosed before the end of 2010 were included in the TMAs used for the present study. Out of the 912 patients included in the cohort, tumor tissue was available from 718 tumors. Two 1-mm cores from each tumor were used for the construction of the TMAs (Beecher, WI, USA). Sections of $4 \mu \mathrm{m}$ were cut and baked on glass slides in a heat chamber for $1 \mathrm{~h}$ at $60^{\circ} \mathrm{C}$. Deparaffination and antigen retrieval was performed using PT Link system (Agilent/Dako A/S). The mouse 


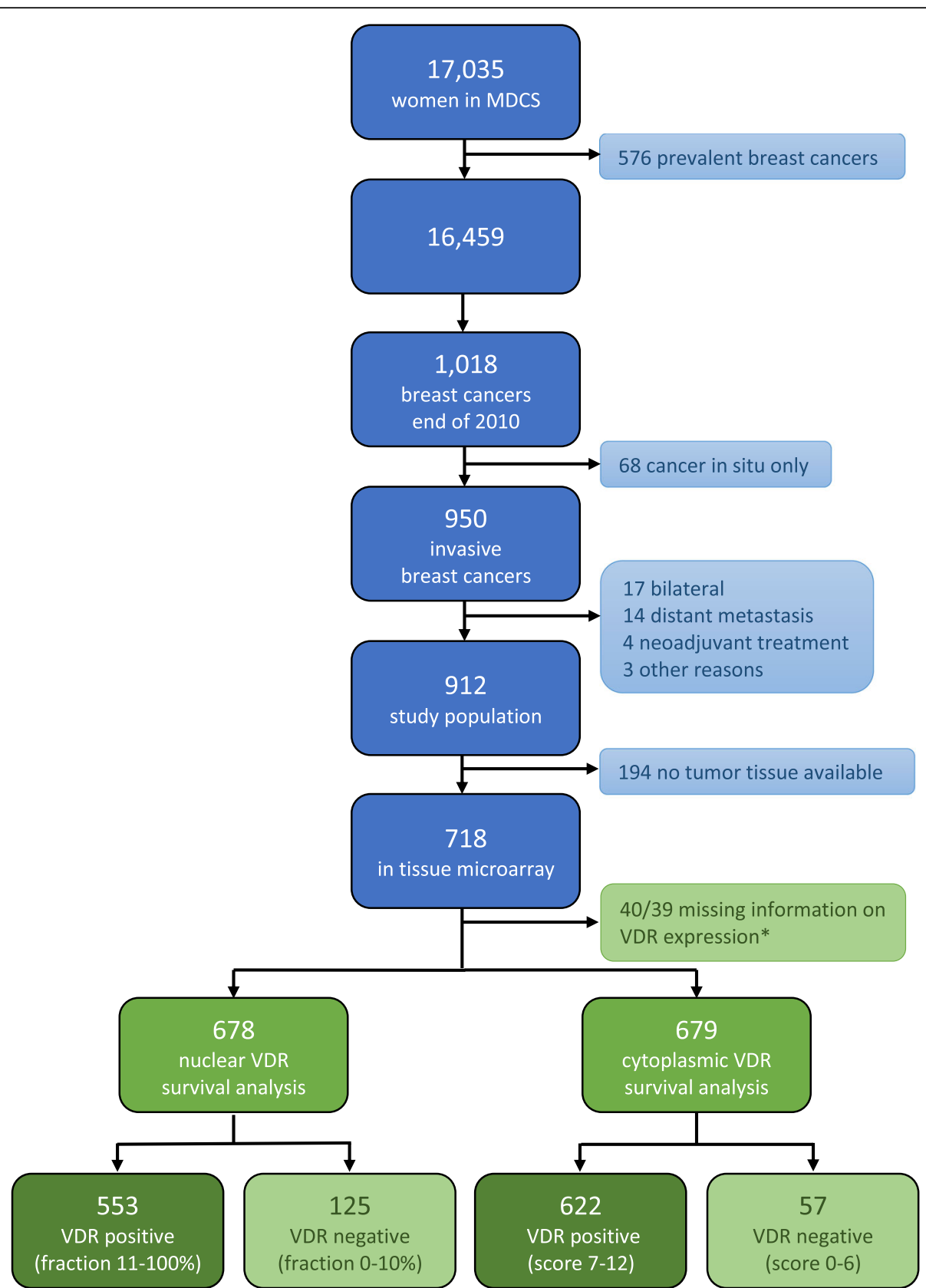

Fig. 1 Flowchart of the study population, inclusion and exclusion. Asterisk indicates that it was not possible to score VDR in the nuclei of 40 core pairs and in the cytoplasm in 39 core pairs

monoclonal D-6 antibody (sc-13133, Santa Cruz Biotechnology) was selected for immunohistochemical analyses of VDR since previous research has found this antibody superior to alternatives as regards to specificity and sensitivity [21, 22]. The antibody was diluted 1:300, and staining was performed automatically in Autostainer Plus (Agilent/Dako A/S), with visualization kit K8010212 (Agilent/Dako A/S) and also counterstained with Mayer's hematoxylin for $2 \mathrm{~min}$. An automated system was used for taking images of the slides, which were thereafter incorporated in the web-based digital pathological platform PathXL Xplore (http://www.pathxl.com, PathXL Ltd., UK). Microscopic evaluations were performed using PathXL, consistently on the same computer screen.

After staining, it was noted that VDR was expressed in several subcellular locations of breast cancer cells (Fig. 2A-H). Staining was observed within the nucleus, in the nuclear membrane, in the cytoplasm, and in the cellular membrane. Semi-quantitative scoring was performed 


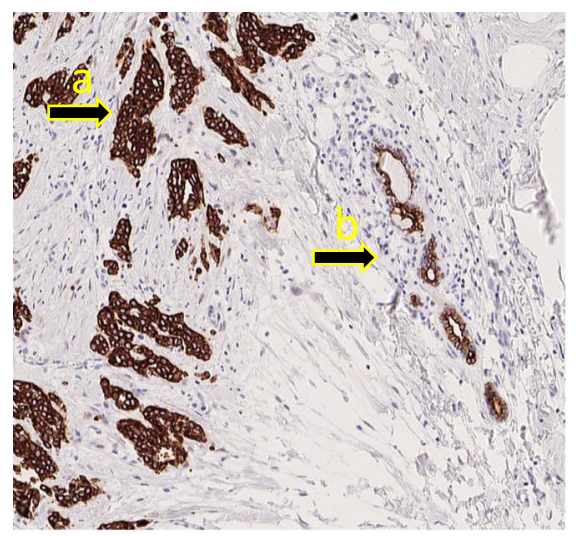

A) Tumor with VDR expression in a) invasive tumor b) cancer in situ

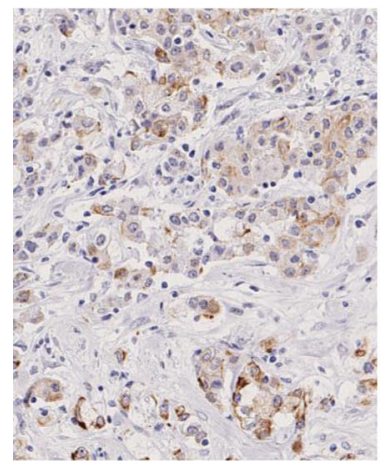

C) Nuclear VDR $0-10 \%$ (neg)

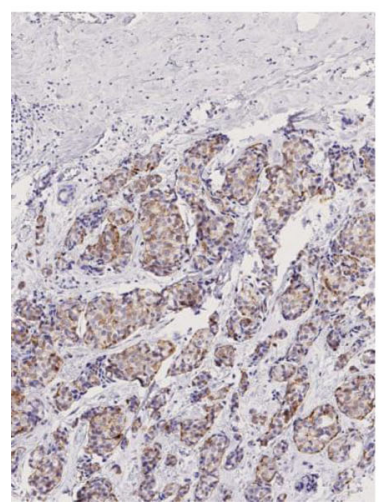

F) Low intensity of cytoplasmic VDR

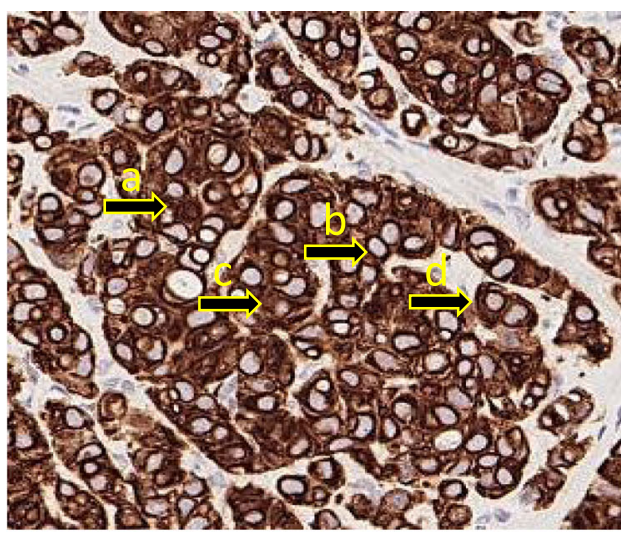

B) Tumor with VDR expression in a) nucleus b) nuclear membrane c) cytoplasm d) cell membrane

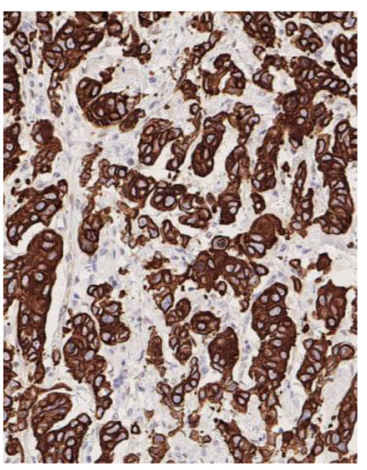

D) Nuclear VDR $11-50 \%$ (pos)

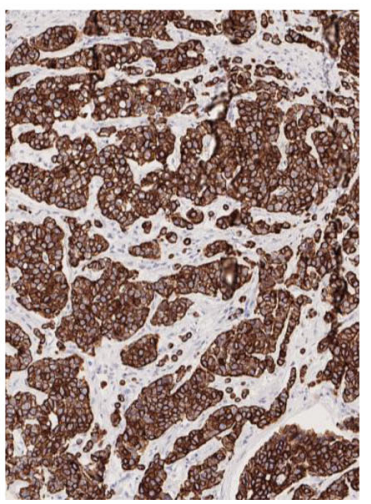

G) Medium intensity of cytoplasmic VDR

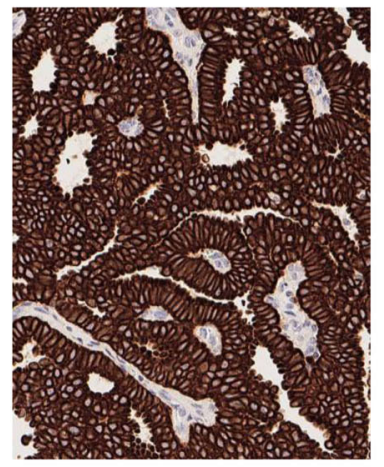

E) Nuclear VDR 51-100\% (pos)

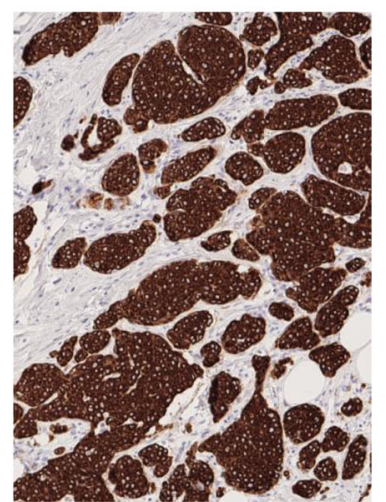

H) High intensity of cytoplasmic VDR

Fig. 2 A-H Examples of VDR expression in tissue microarray

evaluating percentages of positive cells for all locations, $0,1-10 \%, 11-50 \%, 51-75 \%$, and $76-100 \%$. Also, the intensity of staining was evaluated as percentages of highly intense stain in nuclei and nuclear membranes. The intensity of cytoplasmic stain was evaluated on four levels: no stain or low, moderate, or high intensity of stain (Fig. 2G, H).
Each core was scored twice by the same observer, at least 4 weeks apart, blinded to clinical and pathological data but with access to information on invasiveness from the previous evaluation of hematoxylin/ eosin-stained slides of the cores [16].

Due to considerable differences between the first and second readings of VDR scores regarding nuclear 
membrane and cellular membrane, it was decided to focus the statistical analysis on the nuclear and cytoplasmic stain. Discordance of nuclear and cytoplasmic stain between core pairs and different readings was treated as described in Appendix 1. The nuclear stain was defined as negative below a cut-off of $10 \%$, positive $11-50 \%$, and strongly positive above $51 \%$ of stained nuclei. Cytoplasmic score was calculated by multiplying score for fraction, $0(0 \%), 1(1-10 \%)$, $2(11-50 \%), 3(51-75 \%)$, and $4(76-100 \%)$, by intensity scores, 0 (no stain), 1 (low intensity), 2 (moderate intensity), and 3 (high intensity). Cytoplasmic scores were thereafter subdivided into three groups, 0-6, 7-9, and 10-11.

\section{Endpoint retrieval}

All women within the MDCS were followed until December 31, 2016, using the Swedish cause of death registry, which provided information on the date of death, cause of death, and underlying and multiple cause of death. When breast cancer was considered the only cause of death or contributing cause of death, the primary endpoint, breast cancer death, was fulfilled. Other women within the cohort were registered as either dead from other causes, emigrated, or alive. End of follow-up was the date of death, date of emigration, or December 31, 2016.

\section{Statistical analysis}

Tumor characteristics and planned treatment were compared between categories of VDR expression. Distributions were compared using the $\chi^{2}$ test for categorical factors and Kruskal-Wallis test for continuous variables.

Breast cancer mortality (BCM) was calculated as breast cancer-associated death per 100,000 person-years. BCM in relation to VDR expression was tested using a Cox proportional hazards analysis yielding hazard ratios (HRs) with 95\% confidence intervals (CI). The assumption of proportional hazards was met as tested by Kaplan-Meier plots, and statistical significance tested by log-rank tests. The crude model was subsequently adjusted in two multivariate models. Model 2 was adjusted for the season of diagnosis and age at diagnosis which is known to affect levels of vitamin D and might therefore influence the VDR expression. Model 3 was further adjusted for tumor factors known to influence breast cancer prognosis, such as the size of tumor, lymph node status, histological type, and molecular subtypes. As the choice of treatment is heavily dependent on prognostic tumor factors included in the adjusted model, it was decided not to adjust also for treatment variables. All adjusted analyses were first performed as complete cases analyses, only including cases with complete information on all included covariates. Missing data on covariates was thereafter included in the adjusted analyses using a multiple imputation model, described in detail in Appendix 2.
Expression of VDR in association with BCM was tested separately for nuclear fraction and cytoplasmic score, both subdivided in three levels of expression and also two levels of expression (negative vs positive). In a sensitivity analysis, negative nuclear fraction was combined with cytoplasmic score $0-6$, and this combinatory negative VDR score was tested in a Cox proportional hazards analysis for associations with BCM.

Molecular subtypes showed covariance with VDR expression. To investigate whether or not this covariation explained the association found between VDR expression and BCM, the Cox regression model was repeated stratified by molecular subtypes. Since these groups were small and breast cancer death does not occur in some of the subgroups, the analyses were only performed comparing nuclear VDR negativity vs positivity.

SPSS 25.0 (IBM) was used for all statistical analyses.

\section{Results}

\section{Patterns of VDR expression}

VDR was expressed almost exclusively in tumor cells as compared to surrounding cells within the TMA core. When cancer in situ cells were noted in the same core as invasive tumor cells, in situ cells were differently stained compared to invasive cells. Invasive cancer had a more intense stain and a larger fraction of nuclear staining than cancer in situ cells (Fig. 2A). As only invasive cancer cells were scored for this project, such differences were not systematically recorded.

VDR was expressed in all compartments of cancer cells (Fig. 2B). Nuclear VDR expression was assessable in 678 (94.4\%) of tissue core pairs, and cytoplasmic VDR expression as regards to fraction and intensity was scored in 679 (94.6\%) of available tumors. Due to difficulties in distinguishing VDR expression between nuclear membrane and cellular membrane, there was a high percentage of discordance between the first and second rounds of scoring at these compartments $(14.1 \%$ of nuclear membrane fraction and $30.1 \%$ of cellular membrane fraction).

Staining patterns of nuclear VDR fraction are illustrated in Fig. 2C-E. Percentages of highly intense stain in nucleus covaried highly with the fraction of nuclear stain and were therefore not included in further analyses. Distribution of nuclear fraction is presented in Table 1.

The vast majority of tumors 624 (91.9\%) expressed cytoplasmic VDR to a high fraction (76-100\%) of cells. There was a wider distribution of intensity: no stain $(n=7$, $1.0 \%)$, low intensity $(n=26,3.6 \%)$, moderate intensity ( $n=174,24.2 \%)$, and high intensity $(n=472,65.7 \%)$ (Fig. 2F-H). Distribution of scores of cytoplasmic VDR expression is presented in Additional file 1: Table S1. 
Table 1 Distribution of patient and tumor characteristics in relation to nuclear VDR expression

\begin{tabular}{|c|c|c|c|c|c|c|c|}
\hline \multirow{2}{*}{$\begin{array}{l}\text { Eligible cases } \\
\text { Tumor in } \\
\text { tissue microarray } \\
n(\%)\end{array}$} & \multicolumn{7}{|l|}{$\begin{array}{l}\text { All } \\
n=912\end{array}$} \\
\hline & & \multicolumn{5}{|l|}{$\begin{array}{l}\text { Yes } \\
718 \text { (78.7) }\end{array}$} & \multirow[t]{3}{*}{$\begin{array}{l}\text { No } \\
194 \text { (21.3) }\end{array}$} \\
\hline $\begin{array}{l}\text { Nuclear VDR } \\
\text { assessable } \\
n(\%)\end{array}$ & & \multicolumn{4}{|l|}{$\begin{array}{l}\text { Yes } \\
678(94.4)\end{array}$} & \multirow[t]{2}{*}{$\begin{array}{l}\text { No } \\
40(5.6)\end{array}$} & \\
\hline $\begin{array}{l}\text { Nuclear VDR } \\
\text { fraction } \\
n(\%)\end{array}$ & & $\begin{array}{l}\text { Negative } \\
0-10 \% \\
125(18.4)\end{array}$ & $\begin{array}{l}\text { Positive } \\
11-50 \% \\
437(64.5)\end{array}$ & $\begin{array}{l}\text { Positive } \\
51-100 \% \\
116(17.1)\end{array}$ & & & \\
\hline Factor & $\begin{array}{l}n(\%) \text { or } \\
\text { mean (SD) }\end{array}$ & $\begin{array}{l}n(\%) \text { or } \\
\text { mean (SD) }\end{array}$ & $\begin{array}{l}n(\%) \text { or } \\
\text { mean (SD) }\end{array}$ & $\begin{array}{l}n(\%) \text { or } \\
\text { mean (SD) }\end{array}$ & $\begin{array}{l}p \\
\text { value* }^{*}\end{array}$ & $\begin{array}{l}n(\%) \text { or } \\
\text { mean (SD) }\end{array}$ & $\begin{array}{l}n(\%) \text { or } \\
\text { mean (SD) }\end{array}$ \\
\hline Age at baseline & $56.4(7.2)$ & $55.4(7.1)$ & $56.6(7.1)$ & $55.7(7.4)$ & $0.109^{* *}$ & $53.9(7.5)$ & $57.6(7.0)$ \\
\hline Age at diagnosis & $65.4(8.1)$ & $64.1(7.7)$ & $66.0(7.8)$ & $64.8(8.6)$ & $0.026^{* *}$ & $62.1(9.1)$ & $65.9(8.3)$ \\
\hline \multicolumn{8}{|c|}{ Season of diagnosis } \\
\hline Winter & $241(26.4)$ & $35(28.0)$ & $116(26.5)$ & $29(25.0)$ & \multirow[t]{4}{*}{0.646} & $13(32.5)$ & $48(24.7)$ \\
\hline Spring & $221(24.2)$ & $38(30.4)$ & $97(22.2)$ & $27(23.3)$ & & $9(22.5)$ & $50(25.8)$ \\
\hline Summer & $187(20.5)$ & 19 (15.2) & $96(22.0)$ & $23(19.8)$ & & $6(15.0)$ & $43(22.2)$ \\
\hline Fall & $263(28.8)$ & $33(26.4)$ & $128(29.3)$ & 37 (31.9) & & $12(30.0)$ & $53(27.3)$ \\
\hline \multicolumn{8}{|l|}{ BMI at baseline } \\
\hline$<25$ & $467(51.2)$ & $60(48.0)$ & $215(49.2)$ & $70(60.3)$ & \multirow[t]{3}{*}{0.253} & $24(60)$ & $98(50.5)$ \\
\hline$\geq 25-30$ & $310(34)$ & $45(36.0)$ & $150(34.3)$ & $33(28.4)$ & & $11(27.5)$ & $71(36.6)$ \\
\hline$\geq 30$ & $135(14.8)$ & $20(16.0)$ & $72(16.5)$ & $13(11.2)$ & & $5(12.5)$ & $25(12.9)$ \\
\hline \multicolumn{8}{|l|}{ Tumor size } \\
\hline $1-10 \mathrm{~mm}$ & $229(25.8)$ & $14(11.2)$ & $93(21.4)$ & $33(28.7)$ & \multirow[t]{4}{*}{0.002} & $20(50)$ & 69 (39.7) \\
\hline $11-20 \mathrm{~mm}$ & $409(46.1)$ & $57(45.6)$ & $217(49.9)$ & $51(44.3)$ & & $13(32.5)$ & $71(40.8)$ \\
\hline$\geq 21 \mathrm{~mm}$ & $250(28.2)$ & $54(43.2)$ & $125(28.7)$ & $31(27.0)$ & & $6(15.0)$ & $34(19.5)$ \\
\hline Unknown & 24 & 0 & 2 & 1 & & 1 & 20 \\
\hline \multicolumn{8}{|c|}{ Lymph node status } \\
\hline Positive & $262(31.9)$ & $50(41.0)$ & $135(32.8)$ & $36(32.4)$ & \multirow[t]{3}{*}{0.226} & $9(22.5)$ & $32(22.7)$ \\
\hline Negative & $559(68.1)$ & $72(59.0)$ & $276(67.2)$ & 75 (67.6) & & $27(67.5)$ & 109 (77.3) \\
\hline Unknown & 91 & 3 & 26 & 5 & & 4 & 53 \\
\hline \multicolumn{8}{|l|}{ Nottingham grade } \\
\hline 1 & $227(27.2)$ & $7(5.9)$ & $119(27.7)$ & $42(36.5)$ & \multirow[t]{4}{*}{$<0.001$} & $12(30.0)$ & 47 (34.8) \\
\hline$\|$ & $393(47.0)$ & $35(29.4)$ & $219(50.9)$ & $61(53.0)$ & & $16(40.0)$ & $62(45.9)$ \\
\hline III & $216(25.8)$ & $77(64.7)$ & $92(21.4)$ & $12(10.4)$ & & $9(22.5)$ & $26(19.3)$ \\
\hline Unknown & 76 & 6 & 7 & 1 & & 3 & 59 \\
\hline \multicolumn{8}{|l|}{ Histological type } \\
\hline Ductal & $596(70.9)$ & $103(85.1)$ & $290(67.8)$ & $84(72.4)$ & \multirow[t]{4}{*}{$<0.001$} & $24(60.0)$ & $95(68.3)$ \\
\hline Lobular & $166(19.7)$ & $7(5.8)$ & $113(26.4)$ & $20(17.2)$ & & $7(17.5)$ & $19(13.7)$ \\
\hline Other/mixed & $79(9.4)$ & $11(9.1)$ & $25(5.8)$ & $12(10.3)$ & & $6(15.0)$ & $25(18.0)$ \\
\hline Unknown & 71 & 4 & 9 & 0 & & 3 & 55 \\
\hline \multicolumn{8}{|l|}{ ER status } \\
\hline Neg (0-10\%) & $84(10.8)$ & 45 (39.5) & $25(6.1)$ & $4(3.6)$ & \multirow[t]{3}{*}{$<0.001$} & $3(7.5)$ & $7(6.0)$ \\
\hline Pos (> 10\%) & $694(89.2)$ & $69(60.5)$ & 382 (93.9) & $106(96.4)$ & & $28(70.0)$ & $109(94.0)$ \\
\hline Unknown & 134 & 11 & 30 & 6 & & 9 & 78 \\
\hline
\end{tabular}

PgR status 
Table 1 Distribution of patient and tumor characteristics in relation to nuclear VDR expression (Continued)

\begin{tabular}{|c|c|c|c|c|c|c|c|}
\hline \multirow{2}{*}{$\begin{array}{l}\text { Eligible cases } \\
\text { Tumor in } \\
\text { tissue microarray } \\
n(\%)\end{array}$} & \multicolumn{7}{|l|}{$\begin{array}{l}\text { All } \\
n=912\end{array}$} \\
\hline & & \multicolumn{5}{|l|}{$\begin{array}{l}\text { Yes } \\
718 \text { (78.7) }\end{array}$} & \multirow[t]{3}{*}{$\begin{array}{l}\text { No } \\
194(21.3)\end{array}$} \\
\hline $\begin{array}{l}\text { Nuclear VDR } \\
\text { assessable } \\
n(\%)\end{array}$ & & \multicolumn{4}{|l|}{$\begin{array}{l}\text { Yes } \\
678 \text { (94.4) }\end{array}$} & \multirow[t]{2}{*}{$\begin{array}{l}\text { No } \\
40(5.6)\end{array}$} & \\
\hline $\begin{array}{l}\text { Nuclear VDR } \\
\text { fraction } \\
n(\%)\end{array}$ & & $\begin{array}{l}\text { Negative } \\
0-10 \% \\
125(18.4)\end{array}$ & $\begin{array}{l}\text { Positive } \\
11-50 \% \\
437(64.5)\end{array}$ & $\begin{array}{l}\text { Positive } \\
51-100 \% \\
116(17.1)\end{array}$ & & & \\
\hline Factor & $\begin{array}{l}n(\%) \text { or } \\
\text { mean (SD) }\end{array}$ & $\begin{array}{l}n(\%) \text { or } \\
\text { mean (SD) }\end{array}$ & $\begin{array}{l}n(\%) \text { or } \\
\text { mean (SD) }\end{array}$ & $\begin{array}{l}n(\%) \text { or } \\
\text { mean (SD) }\end{array}$ & $\begin{array}{l}p \\
\text { value* }\end{array}$ & $\begin{array}{l}n(\%) \text { or } \\
\text { mean (SD) }\end{array}$ & $\begin{array}{l}n(\%) \text { or } \\
\text { mean (SD) }\end{array}$ \\
\hline Neg (0-10\%) & $311(41.7)$ & $75(67.0)$ & $135(34.5)$ & 39 (36.8) & $<0.001$ & $14(35.0)$ & $48(44.9)$ \\
\hline Pos (> 10\%) & $435(58.3)$ & $37(33.0)$ & $256(65.5)$ & $67(63.2)$ & & $16(40.0)$ & $59(55.1)$ \\
\hline Unknown & 166 & 13 & 46 & 10 & & 10 & 87 \\
\hline \multicolumn{8}{|l|}{ HER2 } \\
\hline Neg & $646(90.9)$ & $102(91.1)$ & 335 (90.3) & $91(91)$ & 0.957 & $26(65.0)$ & $92(92.0)$ \\
\hline Pos & $65(9.1)$ & $10(8.9)$ & $36(9.7)$ & $9(9.0)$ & & $2(5.0)$ & $8(8.0)$ \\
\hline Unknown & 201 & 13 & 66 & 16 & & 12 & 94 \\
\hline \multicolumn{8}{|l|}{ Ki67 } \\
\hline Low & $258(40.6)$ & $16(15.8)$ & $150(45.9)$ & $43(45.7)$ & $<0.001$ & $9(22.5)$ & $40(44.4)$ \\
\hline Intermediate & $198(31.2)$ & $28(27.7)$ & $104(31.8)$ & $36(38.3)$ & & $5(12.5)$ & $25(27.8)$ \\
\hline High & $179(28.2)$ & $57(56.4)$ & $73(22.3)$ & $15(16.0)$ & & $9(22.5)$ & $25(27.8)$ \\
\hline Unknown & 277 & 24 & 110 & 22 & & 17 & 104 \\
\hline \multicolumn{8}{|l|}{ Molecular subtypes } \\
\hline Luminal A-like & $350(55.6)$ & 19 (18.6) & $207(63.1)$ & $62(66.7)$ & $<0.001$ & $11(27.5)$ & $51(62.2)$ \\
\hline Luminal B-like & $158(25.1)$ & $33(32.4)$ & $76(23.2)$ & $20(21.5)$ & & $8(20.0)$ & $21(25.6)$ \\
\hline HER2 positive & $65(10.3)$ & $10(9.8)$ & $36(11.0)$ & $9(9.7)$ & & $2(5.0)$ & $8(9.8)$ \\
\hline Triple negative & $56(8.9)$ & $40(39.2)$ & $9(2.7)$ & $2(2.2)$ & & $3(7.5)$ & $2(2.4)$ \\
\hline Unknown & 283 & 23 & 109 & 23 & & 16 & 112 \\
\hline
\end{tabular}

Percentages do not include missing categories

${ }^{*} p$ values calculated with $x^{2}$ test if not otherwise noted. All $p$ values calculated with only valid categories

**Kruskal-Wallis was used to obtain the $p$ value

\section{Covariation of VDR expression and tumor characteristics}

Distribution of patient and tumor characteristics in relation to nuclear VDR fraction is presented in Table 1. There was a statistically significant covariation between VDR negativity and many tumor characteristics associated with poor prognosis: large tumor size $(p=0.002)$, high Nottingham grade $(p<0.001)$, negative ER status $(p<0.001)$, negative PgR status $(p<0.001)$, and high Ki67 expression $(p<0.001)$. There was also a statistically significant covariation between histological type and VDR expression, where negative tumors more often were considered ductal $(p<0.001)$. When molecular subtypes were compared, it was noted that only $6.6 \%$ of Luminal A-like tumors had a negative VDR expression in the nuclei as compared to $25.6 \%$ among Luminal Blike tumors, and $78.4 \%$ among triple-negative tumors.

A similar pattern was observed when the distribution of patient and tumor characteristics in relation to cytoplasmic VDR score was analyzed (Additional file 1: Table S1). One difference was that cytoplasmic VDR score also showed statistically significant covariation with HER2, as no tumors within the group of low cytoplasmic score $(0-6)$ were considered HER2 positive $(p=0.008)$.

\section{Covariation of VDR expression and breast cancer treatment}

Mastectomies were performed more often on VDR-negative tumors (55\%) compared to VDR-positive tumors 
(41\%). The postoperative treatment conference recommended adjuvant endocrine therapy for a smaller proportion and chemotherapy for a larger proportion of patients with VDR-negative tumors compared to VDR-positive tumors. A similar pattern was seen when cytoplasmic VDR score was compared to treatment factors.

\section{VDR expression in relation to breast cancer mortality}

Mean follow-up was 11.5 years with a standard deviation (SD) of 5.2 years. A Kaplan-Meier analysis confirmed proportional hazards as shown in Fig. 3. Both crude and adjusted analyses showed a statistically significant association between nuclear VDR positivity (a fraction above $10 \%$ of stained nuclei) and a low risk of breast cancer-associated death ( $\mathrm{HR}=0.56,0.34-0.91)$ adjusted analysis) (Table 2). The complete case analysis showed similar but not statistically significant results $(0.61,0.35-1.05)$. Also, similar but not statistically significant results were seen when nuclear VDR fractions $11-50 \%(0.54,0.32-0.89)$ and nuclear VDR fractions $51-100 \%(0.66,0.34-1.28)$ were compared individually to nuclear VDR fraction below $10 \%$. It was also noted that the difference in HR between nuclear VDR fractions $11-50 \%$ and nuclear VDR fractions $51-100 \%$ was small.

As regards to HRs calculated for different cytoplasmic VDR scores, they showed similar results as for nuclear VDR expression, i.e., more VDR expression was associated with decreased risk of breast cancer death, but not statistically significant when adjusted $(0.59,0.30-1.16)$ (Table 2$)$.

\section{VDR expression in relation to breast cancer mortality stratified by molecular subtypes}

HRs for breast cancer death calculated in groups stratified by molecular subtypes are presented in Table 3 . There was a statistically significant association between VDR expression and BCM within the Luminal B-like tumors as nuclear VDR positivity was associated with a decreased risk of breast cancer death $(0.37,0.18-0.77)$. Also, there seemed to be a possible association within the Luminal A-like molecular subtype and reduced risk of breast cancer death, but this association did not reach statistical significance $(0.76,0.32-2.53)$. For HER2-positive and triple-negative molecular subtypes, no statistically significant results were observed.

\section{Discussion}

In the present study, VDR expression was found to be associated with favorable prognostic characteristics, such as small size, low grade, ER positivity, PgR positivity, low Ki67 expression, and Luminal-like molecular subtypes. This corresponds to the finding that VDR-positive tumors were found to be associated with a decreased risk of breast cancer-specific mortality, but this association was also independent of other prognostic factors.

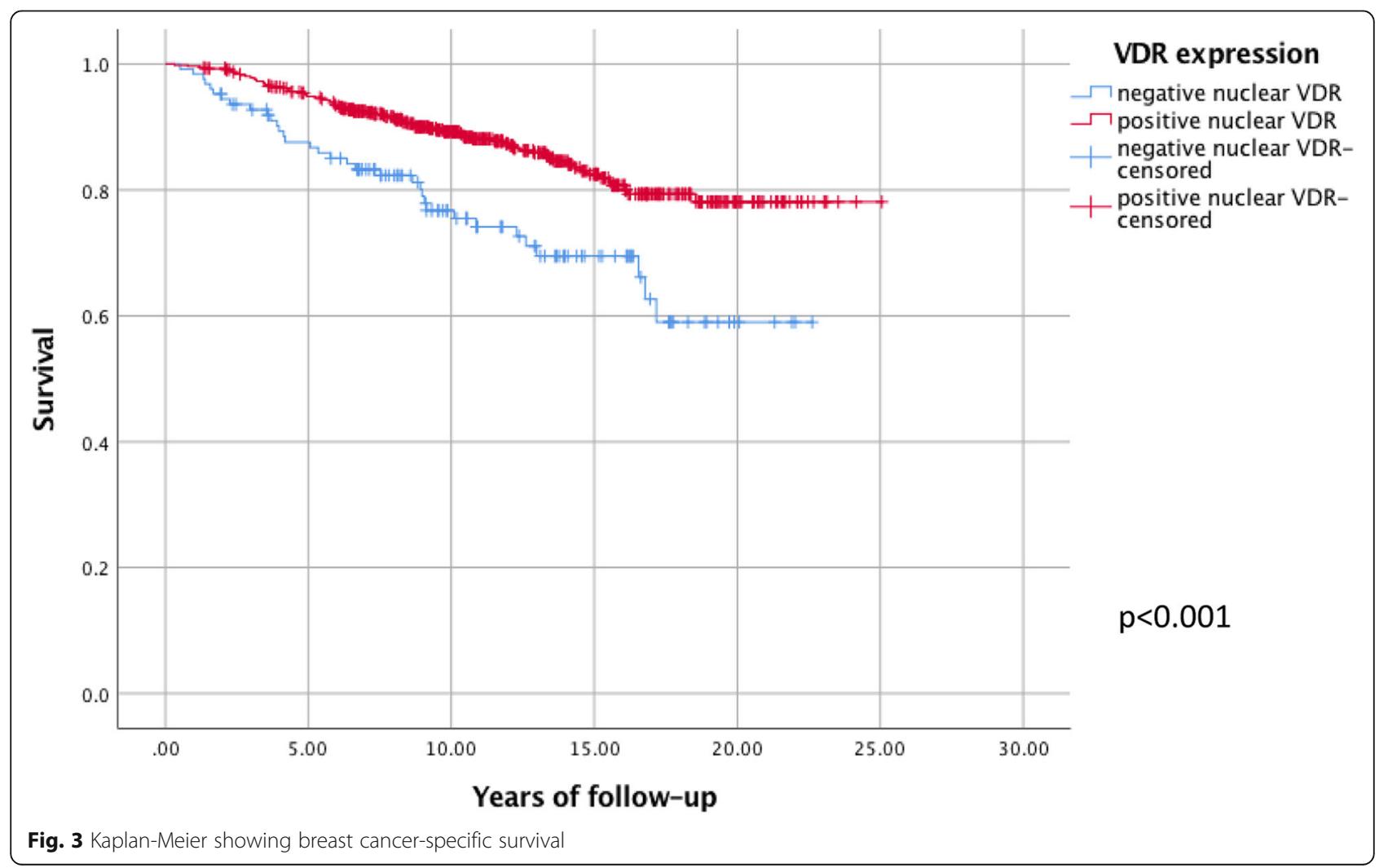


Table 2 Vitamin D receptor expression in relation to breast cancer mortality

\begin{tabular}{|c|c|c|c|c|c|c|c|c|c|}
\hline & & Total $(n)$ & $\begin{array}{l}\text { Person- } \\
\text { years }\end{array}$ & $\begin{array}{l}\text { Dead from } \\
\text { breast cancer }\end{array}$ & $\begin{array}{l}\text { Breast cancer } \\
\text { mortality/100 } 000\end{array}$ & $H R^{a}$ & $H R^{b}$ & $\mathrm{HR}^{\mathrm{c}, \#}$ & $H R^{c_{*} *}$ \\
\hline \multirow[t]{2}{*}{ Nuclear VDR fraction } & $\begin{array}{l}0-10 \% \\
\text { (neg) }\end{array}$ & 125 & 1310 & 34 & 2595 & 1.00 (ref) & 1.00 (ref) & 1.00 (ref) & 1.00 (ref) \\
\hline & $11-100 \%$ (pos) & 553 & 6414 & 77 & 1200 & $\begin{array}{l}0.46(0.31- \\
0.69)\end{array}$ & $\begin{array}{l}0.42(0.28- \\
0.63)\end{array}$ & $\begin{array}{l}0.61(0.35- \\
1.05)\end{array}$ & $\begin{array}{l}0.56(0.34- \\
0.91)\end{array}$ \\
\hline \multirow{3}{*}{$\begin{array}{l}\text { Nuclear VDR } \\
\text { fraction }\end{array}$} & $0-10 \%$ & 125 & 1310 & 34 & 2595 & 1.00 (ref) & 1.00 (ref) & 1.00 (ref) & 1.00 (ref) \\
\hline & $11-50 \%$ & 437 & 5055 & 61 & 1206 & $\begin{array}{l}0.47(0.31- \\
0.71)\end{array}$ & $\begin{array}{l}0.41(0.27- \\
0.63)\end{array}$ & $\begin{array}{l}0.57(0.32- \\
1.01)\end{array}$ & $\begin{array}{l}0.54(0.32- \\
0.89)\end{array}$ \\
\hline & $51-100 \%$ & 116 & 1359 & 16 & 1177 & $\begin{array}{l}0.46(0.25- \\
0.83)\end{array}$ & $\begin{array}{l}0.44(0.24- \\
0.79)\end{array}$ & $\begin{array}{l}0.74(0.35- \\
1.55)\end{array}$ & $\begin{array}{l}0.66(0.34- \\
1.28)\end{array}$ \\
\hline \multirow{2}{*}{$\begin{array}{l}\text { Cytoplasmic } \\
\text { VDR score }\end{array}$} & 0-6 (neg) & 57 & 609 & 17 & 2791 & 1.00 (ref) & 1.00 (ref) & 1.00 (ref) & 1.00 (ref) \\
\hline & 7-12 (pos) & 622 & 7137 & 94 & 1317 & $\begin{array}{l}0.47(0.28- \\
0.79)\end{array}$ & $\begin{array}{l}0.44(0.26- \\
0.74)\end{array}$ & $\begin{array}{l}0.88(0.39- \\
1.97)\end{array}$ & $\begin{array}{l}0.59(0.30- \\
1.16)\end{array}$ \\
\hline \multirow{3}{*}{$\begin{array}{l}\text { Cytoplasmic } \\
\text { VDR score }\end{array}$} & $0-6$ & 57 & 609 & 17 & 2791 & 1.00 (ref) & 1.00 (ref) & 1.00 (ref) & 1.00 (ref) \\
\hline & $7-9$ & 152 & 1712 & 25 & 1460 & $\begin{array}{l}0.52(0.28- \\
0.96)\end{array}$ & $\begin{array}{l}0.50(0.27- \\
0.92)\end{array}$ & $\begin{array}{l}1.01(0.43- \\
2.40)\end{array}$ & $\begin{array}{l}0.58(0.27- \\
1.24)\end{array}$ \\
\hline & $10-12$ & 470 & 5425 & 69 & 1272 & $\begin{array}{l}0.45(0.27- \\
0.77)\end{array}$ & $\begin{array}{l}0.42(0.25- \\
0.72)\end{array}$ & $\begin{array}{l}0.81(0.36- \\
1.86)\end{array}$ & $\begin{array}{l}0.59(0.30- \\
1.18)\end{array}$ \\
\hline
\end{tabular}

${ }^{\text {a Crude analysis }}$

${ }^{\mathrm{b}}$ Adjusted for age at and season of diagnosis

${ }^{c}$ Adjusted for same factors as ${ }^{b}$ but also for size of tumor, lymph node status, histological type, and molecular subtypes

\#Complete case analysis: analysis including only cases with complete information on all covariates

* Multiple imputation performed to include individuals with missing data on covariates in analysis

\section{Immunohistochemistry and patterns of VDR expression} In the present material, VDR was found almost exclusively in tumor cells. As TMA was targeted to evaluate invasive tumors, there was a scarcity of normal breast cells and thorough evaluation of this matter could not be performed. Previous research have found expression of VDR to be higher in in situ and infiltrative carcinoma compared to benign breast disease or normal tissue [23, 24], but others argue the opposite [25].
When this study was initiated, it was expected to find only nuclear staining concerning VDR, since this is what most previous studies have observed $[8,10,26]$. It was therefore surprising to find VDR staining also in the nuclear membrane, the cytoplasm, and the cellular membrane in our TMA. Early research which concluded VDR to be a primary nuclear receptor used radioactive $1,25-(\mathrm{OH}) 2$ vitamin $\mathrm{D}_{3}$ to identify the receptor in mammary cells [8]. More recently, it has been shown that

Table 3 Vitamin D receptor expression in relation to breast cancer mortality stratified by surrogate molecular subtypes

\begin{tabular}{|c|c|c|c|c|c|c|c|c|c|c|c|}
\hline $\begin{array}{l}\text { Surrogate } \\
\text { molecular } \\
\text { subtype }\end{array}$ & $\begin{array}{l}\text { Nuclear } \\
\text { VDR } \\
\text { fraction }\end{array}$ & $n$ & $\begin{array}{l}\text { Person- } \\
\text { years }\end{array}$ & $\begin{array}{l}\text { Dead from } \\
\text { breast cancer }\end{array}$ & $\begin{array}{l}\text { Breast cancer } \\
\text { mortality/100 } 000\end{array}$ & $\mathrm{HR}^{\mathrm{a}}$ & $\mathrm{HR}^{\mathrm{a}, *}$ & $H R^{b}$ & $H R^{b, *}$ & $\mathrm{HR}^{\mathrm{C}}$ & $\mathrm{HR}^{\mathrm{c}, *}$ \\
\hline \multirow[t]{2}{*}{ Luminal A-like } & $0-10 \%$ & 19 & 275 & 4 & 1455 & 1.00 (ref) & 1.00 (ref) & 1.00 (ref) & 1.00 (ref) & 1.00 (ref) & 1.00 (ref) \\
\hline & $11-100 \%$ & 269 & 3132 & 23 & 734 & $\begin{array}{l}0.56(0.19- \\
1.62)\end{array}$ & $\begin{array}{l}0.76(0.27- \\
2.14)\end{array}$ & $\begin{array}{l}0.44(0.15- \\
1.31)\end{array}$ & $\begin{array}{l}0.61(0.21- \\
1.76)\end{array}$ & $\begin{array}{l}0.79(0.24- \\
2.63)^{\mathrm{d}}\end{array}$ & $\begin{array}{l}0.76(0.32- \\
2.53)^{d}\end{array}$ \\
\hline \multirow[t]{2}{*}{ Luminal B-like } & $0-10 \%$ & 33 & 325 & 11 & 3381 & 1.00 (ref) & 1.00 (ref) & 1.00 (ref) & 1.00 (ref) & 1.00 (ref) & 1.00 (ref) \\
\hline & $11-100 \%$ & 96 & 1125 & 15 & 1334 & $\begin{array}{l}0.38(0.18- \\
0.84)\end{array}$ & $\begin{array}{l}0.37(0.18- \\
0.76)\end{array}$ & $\begin{array}{l}0.42(0.19- \\
0.93)\end{array}$ & $\begin{array}{l}0.36(0.17- \\
0.73)\end{array}$ & $\begin{array}{l}0.43(0.19- \\
0.94)^{\mathrm{e}}\end{array}$ & $\begin{array}{l}0.37(0.18- \\
0.77)^{\mathrm{e}}\end{array}$ \\
\hline \multirow[t]{2}{*}{ HER 2 positive } & $0-10 \%$ & 10 & 82 & 3 & 3639 & 1.00 (ref) & 1.00 (ref) & 1.00 (ref) & 1.00 (ref) & 1.00 (ref) & 1.00 (ref) \\
\hline & $11-100 \%$ & 45 & 436 & 12 & 2751 & $\begin{array}{l}0.78(0.22- \\
2.79)\end{array}$ & $\begin{array}{l}0.82(0.25- \\
2.64)\end{array}$ & $\begin{array}{l}0.74(0.20- \\
2.74)\end{array}$ & $\begin{array}{l}0.73(0.22- \\
2.47)\end{array}$ & $\begin{array}{l}1.13(0.31- \\
4.09)^{\mathrm{d}}\end{array}$ & $\begin{array}{l}1.00(0.30- \\
3.33)^{\mathrm{d}}\end{array}$ \\
\hline \multirow[t]{2}{*}{ Triple negative } & $0-10 \%$ & 40 & 419 & 10 & 2388 & 1.00 (ref) & 1.00 (ref) & 1.00 (ref) & 1.00 (ref) & 1.00 (ref) & 1.00 (ref) \\
\hline & $11-100 \%$ & 11 & 123 & 3 & 2440 & $\begin{array}{l}1.04(0.28- \\
3.77)\end{array}$ & $\begin{array}{l}0.85(0.25- \\
2.86)\end{array}$ & $\begin{array}{l}0.80(0.21- \\
3.00)\end{array}$ & $\begin{array}{l}0.70(0.21- \\
2.37)\end{array}$ & $\begin{array}{l}0.87(0.23- \\
3.19)^{\mathrm{e}}\end{array}$ & $\begin{array}{l}0.78(0.23- \\
2.61)^{\mathrm{e}}\end{array}$ \\
\hline
\end{tabular}

${ }^{a}$ Crude analysis

${ }^{\mathrm{b}}$ Adjusted for age at and season of diagnosis

${ }^{c}$ Hazard ratio adjusted for covariate most affecting the estimate: ${ }^{\mathrm{d}}$ lymph node status and ${ }^{\mathrm{e}}$ season of diagnosis

*Multiple imputation performed to include individuals with missing data on molecular subtype 
cytoplasmic unliganded VDR present in tumor cells of cell lines and mouse models promotes cell growth in contrast to the inhibitory effects of intranuclear VDR which has been activated by vitamin D [27]. Another recent study also demonstrated VDR to be localized in the cytoplasm of dividing cells [28]. Hence, unliganded VDR can be found on other locations in the tumor cells, and at least one other study on breast cancer survival has also found VDR in the cytoplasm along with the nuclei [12].

When research on which antibody to use in the study, the Santa-Cruz D-6 antibody was preferred over alternatives, since validation of this particular antibody for immunohistochemistry was considered superior to alternatives [21, 22]. This antibody was not used in the previous studies, which is a possible explanation for different staining patterns.

Since VDR was found on multiple subcellular locations in cancer cells, and there were no previous records in the literature about this, we concluded it best to evaluate the fraction of stained cancer cells at all sites. In an attempt also to score the intensity of nuclear and nuclear membrane VDR expression, an additional score of the highly intensely stained fraction was added. This method was based on previous reports that concluded better reproducibility on very strongly positive scores than including intermediate intensities [29]. Considering intensity in the cytoplasm, it was easier to distinguish intensities of intermediate staining pattern at this location, why it was also included. When evaluating results from scoring, we concluded that scores as regards to nuclear fraction and intensity and cytoplasmic fraction and intensity were congruent enough to be valid.

Scores concerning membranous expression had a low reproducibility and were therefore not included in the statistical analyses. It would have been interesting to compare staining in different subcellular compartments in associations to tumor prognostic factors and breast cancer mortality, since VDR activated by vitamin $\mathrm{D}$ is translocated into the nucleus and has been shown to reduce the viability of triple-negative breast cancer cell lines [30], inhibit breast cancer cell line growth [31], and induce autophagy in breast cancer cell lines and in normal breast tissue of mice [32]. Another previous study has shown that unliganded VDR in the cytoplasm promotes cell growth in contrast to the inhibitory intranuclear ligand-dependent actions of VDR [27]. We suggest that VDR located in the cellular membrane, not activated by vitamin $\mathrm{D}$, hypothetically may be associated with prognostic factors and BCM differently compared to intranuclear VDR. In our study, there were only few individuals with negative nuclear VDR expression and positive cytoplasmic stain, and therefore, we consider that there was not enough power to find any association between this small group of individuals and a possibly elevated risk of BCM. However, tumors with nuclear VDR expression do probably also express VDR in the cytoplasm.

\section{Methodological considerations}

All Swedish residents are given a unique civil registration number at birth or immigration. It is therefore possible to link all women in our cohort to different registries. The Swedish Cause of Death Registry which was used to retrieve information on the cause of death is reported to be virtually complete on the event of death and to $96 \%$ complete to cause of death [33]. Deaths caused by a tumor have been found to be correctly registered in $90 \%$ of cases [34].

Analyses were performed with nuclear fraction and cytoplasmic score analyzed separately (Table 3). As regards to cytoplasmic score, there were few individuals with negative scores $(n=57)$, and results from statistical analyses were harder to interpret, although they seemed to be congruent with results from analyzing nuclear VDR fraction. In a sensitivity analysis, a combinatory score of nuclear and cytoplasmic expression was calculated and used to determine HR of BCM. There were very few individuals with both a negative nuclear fraction and negative cytoplasmic score $(n=54)$, and when this group was expanded to contain either a higher cytoplasmic score (710) or larger nuclear fraction (11-50\%), any effects of VDR negativity could not be observed. Hence, we conclude that in our material a dichotomized variable of a nuclear fraction of VDR expression with a cut-off of $10 \%$ is appropriate to use and will be used in our future studies. Another previous study has also noted that the intensity of VDR expression seems to be of less importance [12].

A tissue microarray (TMA) is not an evaluation of a complete tumor, and therefore, results from an immunohistochemical analysis of TMA are not comparable to a diagnostic immunohistochemical analysis. For research purposes, a TMA is valuable as it makes it possible to evaluate many tumors under a comparably short duration of time. Another weakness of a TMA study is that very small tumors are not represented in the TMA, as seen in Table 1, where small tumors accounted for almost $40 \%$ of tumors not included in TMA but only approximately $20 \%$ of tumors evaluated as regards to nuclear VDR were small.

\section{VDR and associations with prognostic factors and breast cancer treatment}

Tumor factors previously known to predict breast cancer prognosis included in this study was tumor size, lymph node status, tumor grade, histological type, ER and PgR status, HER2 amplification, Ki67 expression, and molecular subtypes derived from the factors above. Covariation of 
statistical significance was seen with most of the prognostic factors, except with lymph node involvement and HER2 amplification. A recent study, which used computer-assisted image analysis for evaluations of nuclear VDR expression, showed very similar results as regards to covariance with tumor prognostic factors [10], which strengthens our results. Earlier, smaller studies have reported divergent results. One study showed associations with tumor size and lymph node involvement, but not with grading, estrogen receptors, progesterone receptors, or HER2 [12]; others reported no associations [13, 14]. The earlier studies were quite small and did not evaluate many of the associations that we found.

As VDR expression covaried with prognostic factors, it was expected to find associations also with the suggested breast cancer treatment. It was noted that VDR-negative tumors more often were surgically treated with mastectomy (they were larger), less often suggested endocrine therapy, and more often chemotherapy (they were ER negative to a larger extent).

\section{VDR and breast cancer mortality (BCM)}

The present study showed a statistically significant association between BCM and VDR tumor expression among breast cancer patients (HR 0.56, 0.34-0.91) (Table 2), which has not been reported previously. Previous studies have not used BCM as endpoint when evaluating breast cancer prognosis [10-14], and associations found between VDR expression and different endpoints are not conclusive. The most recent and largest study, by $\mathrm{Al}$-Azhri et al., reported very similar results to ours as regards to covariation with tumor prognostic factors, but showed no association between VDR expression and overall survival, progression-free survival, or breast cancer-specific survival [10]. Differences in study population such as a shorter follow-up time (mean 72 months, compared to 137 months in our study) and differing tumor characteristics and that they might have included women with metastatic disease in their study population (not mentioned) may explain some of these discrepancies. The earlier and smaller studies have used different approaches on retrieving information on VDR expression and have not associated VDR expression with any differences in survival $[11,13,14]$ although Berger et al. noted that VDR-positive tumors were associated with a longer disease-free interval [11]. Ditsch et al. showed that VDR expression was associated with a between better progression-free survival, and overall survival in univariate analyses [12], which strengthens our results.

Another study which suggested that phenotype of the normal breast tissue surrounding a breast cancer can predict outcome showed that when VDR was expressed along with androgen receptor (AR) and ER in the surrounding breast tissue, patient outcomes were more favorable than when none of those three was expressed [35]. Their results are also in line with ours, suggesting that VDR expression is associated with a better breast cancer prognosis.

Molecular subtypes were included as a covariate in the adjusted model of BCM, which maintained statistical significance when positive nuclear expression (11-100\%) was compared to negative nuclear expression $(0-10 \%)$, although the confidence interval was widened $(0.56$, 0.34-0.91) (Table 2). The model stratified on molecular subtypes showed that for tumors classified as Luminal B, VDR positivity was associated with a decreased BCM. Since breast cancer deaths were uncommon for women with tumors classified as Luminal A, the results had a poor precision and wide confidence intervals. Also, small numbers of HER2-positive tumors and triple-negative tumors made the analyses regarding VDR expression and breast cancer mortality inconclusive. Still, we believe that the stratified model confirmed that the positive prognostic effect seen with positive VDR expression was not all due to covariation with molecular subtypes.

\section{Conclusion}

The present study indicates that high VDR expression in breast cancer cell nuclei is associated with favorable prognostic factors and a decreased risk of breast cancer death. Women with VDR-positive breast tumors have a better breast cancer-specific survival compared to women with VDR-negative tumors. Future studies ought to investigate the combined effect of VDR expression and serum levels of vitamin $\mathrm{D}$ in relation to breast cancer prognosis.

\section{Appendix 1}

Description of handling intraindividual scoring differences Each tumor was represented by two cores in the TMA, and in order to obtain validity of values of expression, scoring was performed twice. At least several weeks, up to months, went between scoring of the same tumor, depending on other obligations of the evaluator (LH). Due to many (seven) scoring categories, an intraindividual discrepancy between the first and second scoring rounds can be expected. Such a discrepancy was treated as follows:

If there were different scores recorded from one core pair in the first evaluation and only one in the second (for example 1,2 and 2,2), the score which was consistent between core pair was recorded. Also, if the score between one core pair differed two steps in one evaluation and the second evaluation showed a score in between (for example 1,3 and 2,2), the second result was recorded. This was considered appropriate since we decided that if there was a discrepancy between cores, the two cores should be evaluated as one entity. When there 
were no differences between core pairs but (only) one score unit between the first and second evaluation, the second score was considered correct, due to the learning curve during the first evaluation. After this procedure, there were still differences as regards to scores for nuclear fraction $(n=30)$, cytoplasmic fraction $(n=18)$, and cytoplasmic intensity $(n=21)$. These tumors were reevaluated, with the pair of cores of the same tumor considered as one entity. After this re-evaluation, score for only four tumors could not be concluded, and a second evaluator (AR) was consulted before the score was determined.

Measurement error due to scoring discrepancies was evaluated by performing sensitivity analyses in which all tumors with any discrepancy (either between cores or between first and second evaluation) between negative and positive nuclear VDR expression were excluded $(n=62)$. These analyses were performed without multiple imputation, and results differed only to a minor extent compared to when the differing cases were included. The adjusted analyses differed the most: 0.65 (0.36-1.15) compared to $0.61(0.35-1.05)$.

\section{Appendix 2}

\section{Description of the imputation model}

Among 718 tumors included in the TMA, there were missing data on the following variables: tumor size $(n=$ $4)$, lymph node status $(n=38)$, tumor grade $(n=17)$, histological type $(n=16)$, ER status $(n=56)$, PgR status $(n=79)$, HER2 expression $(n=107), \mathrm{Ki}-67$ expression $(n=173)$, and molecular subtypes $(n=171)$. Also, it was not possible to evaluate expression of VDR in the cytoplasm for 39 of tumors and in nuclei for 40 of tumors. All above variables were included in the multiple imputation model, except molecular subtypes which were calculated based on results from imputation. Since the analysis in mind for the multiple imputation model was a Cox regression, baseline hazards and breast cancer death were also included in the model, as well as age and season of diagnosis (covariates in the analysis). Further predictors of imputed values and of missingness, such as BMI and year of diagnosis, were also added. SPSS 25.0 (IBM) was used for multiple imputation, and 30 imputations were made. SPSS uses logistic regression for imputation of categorical variables, and linear regression was used for imputation of baseline hazards. Convergence was checked, and it appeared after two iterations, although ten iterations were used to impute values.

In the Cox analysis only, imputed values for covariates (tumor size, lymph node status, histological type, and molecular subtypes) were used. Only tumors with values based on defined scores of VDR expression were included in the final analyses.

\section{Additional file}

Additional file 1: Distribution of patient and tumor characteristics in relation to cytoplasmic VDR expression. (DOCX $24 \mathrm{~kb}$ )

\section{Abbreviations}

BCM: Breast cancer mortality; Cl: Confidence interval; ER: Estrogen receptor; HER2: Human epidermal growth factor-2; HR: Hazard ratio; ISH: In situ hybridization; Ki67: Proliferation index factor Ki67; MDCS: Malmö Diet and Cancer Study; PgR: Progesterone receptor; SD: Standard deviation; TMA: Tissue microarray; VDR: Vitamin D receptor

\section{Acknowledgements}

The authors wish to thank registered nurse and data manager Anna Hwasser, who prepared the original files and assisted in the data management; biomedical analysist Björn Nodin, who constructed TMAs; and research engineer Kristina Lövgren, who prepared and stained TMA slides for VDR.

\section{Authors' contributions}

LH scored the VDR expression and did all the statistical analyses. LH was also the main author of the manuscript and constructed the tables and figures. STB, AR, and JM supervised the project. SB supervised the pathological evaluation. KE evaluated the inclusion criteria of TMA and revised the manuscript. MS provided the information on molecular subtypes and revised the manuscript. All authors read and approved the final manuscript.

\section{Funding}

The present study was supported by the Swedish Cancer Society, The Gunnar Nilsson Cancer Foundation, The Henning and Ida Persson Foundation, The Einar and Inga Nilsson Foundation, The Malmö University Hospital Cancer Research Fund, The Skåne University Hospital Funds and Donations, and The Region Skåne (ALF).

\section{Availability of data and materials}

The data that support the findings of this study are available on request from the corresponding author $(\mathrm{LH})$. The data are not publicly available due to Swedish restrictions.

Ethics approval and consent to participate

The MDCS and the present study were approved by the ethics committee in Lund, Sweden, MDCS (LU 51-90) and the present study (Dnr 652/2005 and Dnr 23/2007).

Written informed consent was obtained from all study participants on the inclusion of MDCS. Follow-up studies have been advertised in local media, with information about an option to withdraw.

Consent for publication

Not applicable.

\section{Competing interests}

The authors declare that they have no competing interests.

\section{Author details}

${ }^{1}$ Department of Surgery, Lund University, Skåne University Hospital, SE-205 02 Malmö, Sweden. '2Department of Oncology, Aarhus University, Aarhus University Hospital, DE-8000 Aarhus C, Denmark. ${ }^{3}$ Division of Oncology and Pathology, Department of Clinical Sciences, Lund University, Skåne University Hospital, SE-221 85 Lund, Sweden.

Received: 12 February 2019 Accepted: 15 July 2019

Published online: 29 July 2019

\section{References}

1. Yao S, Kwan ML, Ergas IJ, Roh JM, Cheng TD, Hong CC, McCann SE, Tang L, Davis W, Liu S, et al. Association of serum level of vitamin D at diagnosis with breast cancer survival: a case-cohort analysis in the pathways study. JAMA Oncol. 2017;3(3):351-7. 
2. Jacobs ET, Kohler LN, Kunihiro AG, Jurutka PW. Vitamin D and colorectal, breast, and prostate cancers: a review of the epidemiological evidence. J Cancer. 2016;7(3):232-40.

3. Freedman DM, Looker AC, Chang SC, Graubard BI. Prospective study of serum vitamin D and cancer mortality in the United States. J Natl Cancer Inst. 2007:99(21):1594-602.

4. Rose AA, Elser C, Ennis M, Goodwin PJ. Blood levels of vitamin D and early stage breast cancer prognosis: a systematic review and meta-analysis. Breast Cancer Res Treat. 2013;141(3):331-9.

5. Huss L, Butt S, Borgquist S, Almquist M, Malm J, Manjer J. Serum levels of vitamin $\mathrm{D}$, parathyroid hormone and calcium in relation to survival following breast cancer. Cancer Causes Control. 2014;25(9):1131-40.

6. Haussler MR, Whitfield GK, Haussler CA, Hsieh J-C, Thompson PD, Selznick SH, Dominguez CE, Jurutka PW. The nuclear vitamin D receptor: biological and molecular regulatory properties revealed. J Bone Miner Res. 1998;13(3): 325-49

7. Berger U, Wilson P, McClelland RA, Colston K, Haussler MR, Pike JW, Coombes RC. Immunocytochemical detection of 1,25-dihydroxyvitamin D receptors in normal human tissues. J Clin Endocrinol Metab. 1988; 67(3):607-13.

8. Narbaitz R, Sar M, Stumpf WE, Huang S, DeLuca HF. 1,25-Dihydroxyvitamin D3 target cells in rat mammary gland. Horm Res. 1981;15(4):263-9.

9. Friedrich M, Axt-Fliedner R, Villena-Heinsen C, Tilgen W, Schmidt W, Reichrath J. Analysis of vitamin D-receptor (VDR) and retinoid X-receptor alpha in breast cancer. Histochem J. 2002;34(1-2):35-40.

10. Al-Azhri J, Zhang Y, Bshara W, Zirpoli GR, McCann SE, Khoury T, Morrison CD, Edge SB, Ambrosone CB, Yao S. Tumor expression of vitamin D receptor and breast cancer histopathological characteristics and prognosis. Clin Cancer Res. 2017;23(1):97-103.

11. Berger U, McClelland RA, Wilson P, Greene GL, Haussler MR, Pike JW, Colston K, Easton D, Coombes RC. Immunocytochemical determination of estrogen receptor, progesterone receptor, and 1,25-dihydroxyvitamin D3 receptor in breast cancer and relationship to prognosis. Cancer Res. 1991; 51(1):239-44.

12. Ditsch N, Toth B, Mayr D, Lenhard M, Gallwas J, Weissenbacher T, Dannecker $C$, Friese $\mathrm{K}$, Jeschke $\mathrm{U}$. The association between vitamin $\mathrm{D}$ receptor expression and prolonged overall survival in breast cancer. J Histochem Cytochem. 2012:60(2):121-9.

13. Eisman JA, Suva LJ, Martin TJ. Significance of 1,25-dihydroxyvitamin D3 receptor in primary breast cancers. Cancer Res. 1986;46(10):5406-8.

14. Freake HC, Abeyasekera G, Iwasaki J, Marcocci C, Maclntyre I, McClelland RA, Skilton RA, Easton DF, Coombes RC. Measurement of 1,25-dihydroxyvitamin D3 receptors in breast cancer and their relationship to biochemical and clinical indices. Cancer Res. 1984:44(4):1677-81.

15. Friedrich M, Villena-Heinsen C, Tilgen W, Schmidt W, Reichrat J, Axt-Fliedner R. Vitamin D receptor (VDR) expression is not a prognostic factor in breast cancer. Anticancer Res. 2002;22(3):1919-24.

16. Elebro K, Bendahl PO, Jernstrom H, Borgquist S. Androgen receptor expression and breast cancer mortality in a population-based prospective cohort. Breast Cancer Res Treat. 2017;165(3):645-57.

17. Borgquist S, Anagnostaki L, Jirstrom K, Landberg G, Manjer J. Breast tumours following combined hormone replacement therapy express favourable prognostic factors. Int J Cancer. 2007;120(10):2202-7.

18. Butt S: Reproductive factors and breast cancer - parity, breastfeeding and genetic predisposition in relation to risk and prognosis. Lund University, Faculty of Medicine Doctoral Dissertation Series, 2011:10:

19. Borgquist S, Djerbi S, Ponten F, Anagnostaki L, Goldman M, Gaber A, Manjer J, Landberg G, Jirstrom K. HMG-CoA reductase expression in breast cancer is associated with a less aggressive phenotype and influenced by anthropometric factors. Int J Cancer. 2008;123(5):1146-53.

20. Guidelines for surgical and oncologic treatment of breast cancer - regional adaption of the national treatment guidelines, Region West and South [In Swedish: Lathund för kirurgisk och onkologisk behandling av bröstcancer Regional anpassning av nationellt vårdprogram, Region Väst och Syd]. [https://www.cancercentrum.se/globalassets/cancerdiagnoser/brost/syd/ sydsvenska-brostcancergruppens-lathund-170401-180331.pdf.]

21. Costa PL, Franca MM, Ferraz-de-Souza B. Nonspecific binding of a frequently used vitamin D receptor (VDR) antibody: important implications for vitamin D research in human health. Endocrine. 2016;54(2):556-9.
22. Wang Y, Becklund BR, DeLuca HF. Identification of a highly specific and versatile vitamin D receptor antibody. Arch Biochem Biophys. 2010; 494(2):166-77.

23. Conde I, Paniagua R, Fraile B, Ruiz A, Arenas MI. Expression of vitamin D3 receptor and retinoid receptors in human breast cancer: identification of potential heterodimeric receptors. Int J Oncol. 2004;25(4):1183-91.

24. Zhalehjoo N, Shakiba Y, Panjehpour M. Alterations of vitamin D receptor (VDR) expression profile in normal and malignant breast tissues. Clin Lab. 2016;62(8):1461-8.

25. Lopes N, Sousa B, Martins D, Gomes M, Vieira D, Veronese LA, Milanezi F, Paredes J, Costa Jé L, Schmitt F. Alterations in vitamin D signalling and metabolic pathways in breast cancer progression: a study of VDR, CYP27B1 and CYP24A1 expression in benign and malignant breast lesions vitamin D pathways unbalanced in breast lesions. BMC Cancer. 2010;10:483.

26. Berger U, Wilson P, McClelland RA, Colston K, Haussler MR, Pike JW, Coombes RC. Immunocytochemical detection of 1,25-dihydroxyvitamin D3 receptor in breast cancer. Cancer Res. 1987;47(24 Pt 1):6793-9.

27. Trivedi T, Zheng Y, Fournier PGJ, Murthy S, John S, Schillo S, Dunstan CR, Mohammad KS, Zhou H, Seibel MJ, et al. The vitamin D receptor is involved in the regulation of human breast cancer cell growth via a ligandindependent function in cytoplasm. Oncotarget. 2017;8(16):26687-701.

28. Cui X, Pertile R, Eyles DW. The vitamin D receptor (VDR) binds to the nuclear matrix via its hinge domain: a potential mechanism for the reduction in VDR mediated transcription in mitotic cells. Mol Cell Endocrinol. 2018;472:18-25.

29. van Diest PJ, van Dam P, Henzen-Logmans SC, Berns E, van der Burg ME, Green J, Vergote I. A scoring system for immunohistochemical staining: consensus report of the task force for basic research of the EORTC-GCCG. European Organization for Research and Treatment of CancerGynaecological Cancer Cooperative Group. J Clin Pathol. 1997;50(10):801-4.

30. Thakkar A, Wang B, Picon-Ruiz M, Buchwald P, Ince TA. Vitamin D and androgen receptor-targeted therapy for triple-negative breast cancer. Breast Cancer Res Treat. 2016:157(1):77-90.

31. Murray A, Madden SF, Synnott NC, Klinger R, O'Connor D, O'Donovan N, Gallagher W, Crown J, Duffy MJ. Vitamin D receptor as a target for breast cancer therapy. Endocr Relat Cancer. 2017;24(4):181-95.

32. Tavera-Mendoza LE, Westerling T, Libby E, Marusyk A, Cato L, Cassani R, Cameron LA, Ficarro SB, Marto JA, Klawitter J, et al. Vitamin D receptor regulates autophagy in the normal mammary gland and in luminal breast cancer cells. Proc Natl Acad Sci U S A. 2017;114(11):E2186-e2194.

33. Brooke HL, Talback M, Hornblad J, Johansson LA, Ludvigsson JF, Druid H, Feychting M, Ljung R. The Swedish cause of death register. Eur J Epidemiol. 2017;32(9):765-73.

34. Johansson LA, Bjorkenstam C, Westerling R. Unexplained differences between hospital and mortality data indicated mistakes in death certification: an investigation of 1,094 deaths in Sweden during 1995. J Clin Epidemiol. 2009;62(11):1202-9.

35. Santagata S, Thakkar A, Ergonul A, Wang B, Woo T, Hu R, Harrell JC, McNamara G, Schwede M, Culhane AC, et al. Taxonomy of breast cancer based on normal cell phenotype predicts outcome. J Clin Invest. 2014; 124(2):859-70.

\section{Publisher's Note}

Springer Nature remains neutral with regard to jurisdictional claims in published maps and institutional affiliations.

Ready to submit your research? Choose BMC and benefit from:

- fast, convenient online submission

- thorough peer review by experienced researchers in your field

- rapid publication on acceptance

- support for research data, including large and complex data types

- gold Open Access which fosters wider collaboration and increased citations

- maximum visibility for your research: over $100 \mathrm{M}$ website views per year

At $\mathrm{BMC}$, research is always in progress.

Learn more biomedcentral.com/submissions 